\title{
Itinéraires Itinéraires
}

Littérature, textes, cultures

\section{Béatrice Jongy, Yves Chevrel et Véronique Léonard-Roques (dir.), Le Fils prodigue et les siens, $\mathrm{XX} \mathrm{X}^{e}-\mathrm{XX} \mathrm{I}^{e}$ siècles}

\section{Lydie Laroque}

\section{(2) OpenEdition}

\section{Journals}

\section{Édition électronique}

URL : http://journals.openedition.org/itineraires/2142

DOI : $10.4000 /$ itineraires. 2142

ISSN : 2427-920X

Éditeur

Pléiade

\section{Édition imprimée}

Date de publication : 1 mai 2010

Pagination : 190-194

ISBN : 978-2-296-11224-7

ISSN : $2100-1340$

\section{Référence électronique}

Lydie Laroque, «Béatrice Jongy, Yves Chevrel et Véronique Léonard-Roques (dir.), Le Fils prodigue et les siens, $x x^{e}-\left.x x\right|^{e}$ siècles », Itinéraires [En ligne], 2010-1 | 2010, mis en ligne le 01 mai 2010, consulté le 22 septembre 2020. URL : http://journals.openedition.org/itineraires/2142 ; DOI : https://doi.org/ 10.4000/itineraires.2142

\section{Ce document a été généré automatiquement le 22 septembre 2020}

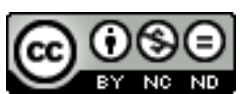

Itinéraires est mis à disposition selon les termes de la licence Creative Commons Attribution - Pas d'Utilisation Commerciale - Pas de Modification 4.0 International. 


\title{
Béatrice Jongy, Yves Chevrel et Véronique Léonard-Roques (dir.), Le Fils prodigue et les siens, $\mathrm{XX}^{e}-\mathrm{XXI}{ }^{e}$ siècles
}

\author{
Lydie Laroque
}

\section{RÉFÉRENCE}

Béatrice Jongy, Yves Chevrel et Véronique Léonard-Roques (dir.), Le Fils prodigue et les siens, $\mathrm{XX}^{e}-\mathrm{XXI} \mathrm{I}^{e}$ siècles, Paris, Le Cerf, 2009, $308 \mathrm{p}$.

1 Béatrice Jongy s'interroge sur la parabole du Fils prodigue, figure biblique prégnante de la littérature et de l'art contemporains, en compagnie de deux spécialistes des relations entre Bible et littérature, Véronique Léonard-Roques et Yves Chevrel - qui codirigent le recueil - mais également de douze autres chercheurs. Elle part de l'hypothèse que le Fils prodigue s'est transfiguré après être tombé dans l'oubli au XIX ${ }^{e}$ siècle, pour resurgir en tant que reflet du monde contemporain.

L'ouverture de l'ouvrage collectif rappelle le schéma originel de l'épisode biblique ainsi que son évolution historique, tant sur le plan exégétique que sur le plan littéraire ou psychanalytique. Yves Chevrel, dans son article, «Solitaire parmi les siens », souligne la simplicité de cette parabole orientale à trois personnages rapportée par Luc $(15,11-32)$. Celle-ci soulève néanmoins beaucoup de questions. C'est le fils cadet qui a retenu de manière presque exclusive l'attention de ceux qui ont traité cette figure en Europe à partir du Moyen Âge, période où commence à se mettre en place une "mythisation » avant tout religieuse. Mais dès le XIX ${ }^{e}$ siècle, à partir du poème de Mallarmé, et surtout au $\mathrm{Xx}^{\mathrm{e}}$, avec Gide (Le Retour de l'enfant prodigue), Rilke (Aufzeichnungen des Malte Laurids Brigge) et Kafka (Heimkehr), les Fils prodigues deviennent les représentants de la Modernité. Aujourd'hui, il est difficile de les identifier car ils ne sont plus ni fils ni prodigues et renvoient, dans une famille étouffante, à une grave crise de l'identité personnelle. À ce panorama de l'histoire littéraire, Elian Cuvillier ajoute, dans « La 
parabole d'un père et de ses deux fils, une exégèse de Luc $(15,11-32)$ », un historique des interprétations (exégétiques ou psychanalytiques) de la parabole. Il souligne tout d'abord un premier niveau d'interprétation par les évangiles qui perdent le contexte originel : la parabole du Fils prodigue est « une métaphore étendue en récit » (p. 37) qui joue sur la surprise. Cuvillier retrace ensuite l'évolution exégétique à partir du texte de Luc : jusqu'au XIX ${ }^{e}$ siècle, c'est la lecture allégorique qui domine. Au début du XIX siècle cependant, Adolf Jülicher ouvre de nouvelles voies interprétatives. Deux courants se détachent depuis bientôt deux mille ans : le premier fait de la parabole une mise en scène de l'histoire du salut de l'individu ou du groupe. Le second l'interprète comme une proclamation de la miséricorde et de l'amour de Dieu pour les pécheurs. Mais depuis une quarantaine d'années, les lectures psychanalytiques se multiplient également, interrogeant les relations entre le père et ses deux fils indépendamment des valeurs théologiques habituelles.

3 La deuxième partie de l'ouvrage s'intéresse plus spécifiquement à la littérature des $\mathrm{xx}^{\mathrm{e}}$ et $\mathrm{xxI}^{\mathrm{e}}$ siècles, qui a repris la parabole biblique. Elle met en évidence les rapprochements du Fils prodigue avec d'autres figures mythiques et la spécificité des réécritures propres à des auteurs particuliers ou à différentes cultures. Ainsi, Tatiana Victoroff pointe l'originalité du traitement de la parabole par les écrivains russes: ceux-ci mettent l'accent sur l'amour du père envers son fils plus que sur la perversion $\mathrm{du}$ rapport père-fils privilégiée par la littérature occidentale. Ces écrivains y voient également «dans ce siècle de bouleversement, l'histoire de tout un peuple» (p. 75). La littérature belge contemporaine, quant à elle, rejoint certaines interprétations de Gide et Rilke, ainsi que le remarque Katherine Rondou dans «Panorama de la parabole de l'enfant prodigue dans la littérature belge des $\mathrm{xx}^{\mathrm{e}}$ et $\mathrm{xxI}^{\mathrm{e}}$ siècles ». Mais la Flandre offre également une lecture spécifique : « une mise en garde moralisatrice » (p. 78).

Les deux articles suivants cernent plus précisément le motif du Fils prodigue par le rapprochement et la comparaison de deux écrivains. La réflexion sur l'enfant prodigue peut garder un ancrage liturgique. C'est ce que constate Dominique Millet-Gérard avec «Exégèse symbolique et poétique de l'enfant prodigue chez Bloy et Claudel ». Bloy s'inscrit en porte-à-faux contre les interprétations courantes en prenant le parti des aînés lésés. La parabole devient une sorte de compendium d'enseignement christique chez Claudel. À côté de ces passages grandioses, la verve des deux auteurs actualise la parabole par le mélange de styles élevés et bas. Les écrivains en dégagent ainsi une théorie esthétique et spirituelle : ils voient dans le genre même de la parabole le patron analogique d'un art qui combine un réalisme de la simplicité et une ambition spirituelle. Si Bloy et Claudel se rejoignent dans une visée commune - la réhabilitation de l'art chrétien - Hermann Hesse et José Pliya se rapprochent dans la mesure où ils croisent tous deux le thème du Fils prodigue avec le mythe de Caïn. Mais Véronique Leonard-Roques montre aussi, dans «Fils perdu et fils fidèle au miroir du mythe de Caïn ", qu'au début $\mathrm{du} x \mathrm{x}^{\mathrm{e}}$ siècle, cette coalescence sert la quête et l'espoir d'un renouvellement socioculturel. À l'aube du xxI ${ }^{e}$ siècle, elle traduit les incertitudes et les doutes propres à la condition postmoderne.

Les articles suivants centrent leur propos sur la spécificité de certains écrivains qui ont repris la figure du Fils prodigue et l'ont fait entrer dans la Modernité. Au carrefour des enjeux intimes et des enjeux esthétiques, le poème "Der Auszug des verlorenen Sohnes " révèle la radicalité des refus de Rilke (son rejet du christianisme) autant que la conscience de la voie à suivre pour parvenir à la maîtrise de soi et de son art. Comme 
Jean-Yves Masson le constate dans son article, Rilke n'est pas absolument opposé au message évangélique mais il le neutralise en refusant toute condamnation morale de celui qui part. Il s'agit pour lui de clarifier sa position face à l'héritage : le chemin juste passe "à égale distance des stratégies de la rupture et des partis pris conservateurs " (p.150). Le poète polonais Tadeusz Różewicz va plus loin en faisant éclater les structures de la parabole biblique pour la contester et traduire l'inquiétude métaphysique de l'homme au sortir de la guerre. Mais selon Danièle Chauvin, dans « Il ne faut pas revenir. Le Fils prodigue de Tadeusz Różewicz ", cette parabole est aussi " celle d'un poète qui ne trouve plus sa place en un siècle barbare et ne peut exister qu'entre deux battements de porte»(p.166). D'après l'écrivain autrichien Soma Morgenstern, il n'y a pas non plus de retour possible pour le Fils prodigue. Cependant, en acceptant de reconsidérer le réel sous un jour différent, Morgenstern suit à la fois le message de la parabole et celui de l'appel de la Teshuva. Christian Mariotte montre ainsi que Morgenstern est confronté aux conflits meurtriers de son époque mais qu'il insiste en même temps sur la profondeur de sa foi «en insérant la parabole néotestamentaire dans un contexte spécifiquement juif » (p. 174). À l'inverse, c'est au sein d'un univers désacralisé que se déroule le roman de Sinclair Lewis, réécriture américaine inversée de la parabole évangélique. Sylvie Parizet explique dans «La parabole inversée. Le cas des parents prodigues de Sinclair Lewis » que The Prodigal Parents exprime " la sourde violence que revêt l'accès à l'autonomie en un monde qui ne possède plus d'autres valeurs que l'argent » (p. 203), et que les pères ont déserté. Le motif de la fuite, qui ressortit du poids des liens familiaux, se retrouve de même associé à la négation de la singularité par la famille, pour le dramaturge français Jean-Luc Lagarce. Béatrice Jongy montre, dans « Le fils prodigue en écrivain : Jean-Luc Lagarce : Juste la fin du monde ", que le Fils prodigue revient cette fois dans l'espoir de se faire connaître; mais il n'échappe pas à l'identité assignée par sa famille et meurt. Néanmoins il triomphe car il escamote sa chair pour se transformer en livre, « corps libre » (p. 224).

6 La dernière partie de l'ouvrage élargit le propos aux réécritures artistiques et médiatiques de la parabole. Le Fils prodigue semble plus sage, tout au moins dans les années 1950 et 1960, où la dimension morale et spirituelle prédomine. Le britannique Britten met d'emblée l'opéra sous le signe de la religion catholique, comme le remarque Élisabeth Rallo Ditche dans "Le Fils prodigue de Benjamin Britten». Le cinéma hollywoodien, quant à lui, fait une lecture manichéenne de la parabole et exalte le monothéisme. Gaël Prigent considère dans son article, «Le Fils prodigue (The Prodigal, 1955) de Richard Thorpe: Un retour à la Bible?» que le film s'apparente à une relecture de l'épisode à l'aune de la morale et des valeurs américaines de l'après Seconde Guerre mondiale. Cependant, un demi-siècle plus tard, le Fils prodigue se révolte : la dimension religieuse disparaît. Comme en littérature, la figure du père est remise en cause, ainsi que le constate Benjamin Thomas à propos d'une variation offerte au début $\mathrm{du} \mathrm{xxI}^{\mathrm{e}}$ siècle par un jeune cinéaste russe, Le Retour d'Andreï Zviaguintsev. Le Fils prodigue a opéré un retournement de situation car «c'est lui qui juge l'héritage qu'a à lui proposer le père » (p. 265). À la télévision, l'histoire du Fils prodigue finit mal. Pour Joseph Belletante, le Fils prodigue, dans la série américaine Six feet under d'Alan Ball, devient adulte et s'affranchit de l'autorité du père miséricordieux, « en évoluant vers son autonomie avec le réel pour seule contrainte » (p. 280). 
7 L'ouvrage se clôt sur une postface de Béatrice Jongy qui dégage les lignes dominantes de l'interprétation contemporaine du Fils prodigue. Le thème, aux $\mathrm{xx}^{\mathrm{e}}$ et $\mathrm{xxI}^{\mathrm{e}}$ siècles, s'inscrit dans la crise du sujet. Les réécritures de la parabole, élargie à la relation parents-enfants, mettent en scène un amour qui assujettit à la fois celui qui l'éprouve et son objet. L'enfant prodigue cherche dès lors à se libérer. Il renonce au dialogue, préférant le départ à un amour pervers. Mais il ressent aussi la mouvance de son identité et revient pour mourir ou pour constater sa solitude. Par ailleurs, le poème de Rilke semble être le point central d'une galaxie de réécritures.

8 Malgré une grande variété de textes, on regrettera peut-être l'absence d'études consacrées aux réécritures majeures de Kafka et de Gide, ainsi que la part restreinte d'articles centrés sur le croisement du Fils prodigue avec d'autres figures bibliques: outre Caïn, Jonas ou Job sont aussi des échos à l'angoissante question de l'élection et de l'injustice. Cependant, cet ouvrage collectif a le mérite de mettre en avant la modernité de la parabole, en l'associant à des considérations psychanalytiques et ontologiques : le Fils prodigue a connu des métamorphoses et s'adapte désormais aux interrogations contemporaines. Le recueil offre également un large éventail de réécritures littéraires et de relectures artistiques ou médiatiques (opéra, cinéma, télévision) représentatives de cultures diverses. Grâce à cet élargissement du propos l'ouvrage peut toucher un large public et dépasser le cadre restreint de l'université. Il montre comment le Fils prodigue s'invite désormais dans notre vie quotidienne et concerne donc tout lecteur cultivé.

\section{AUTEURS}

\section{LYDIE LAROQUE}

IUFM de Versailles 